\title{
Experimental Studies and Modeling of Complete H-Mode Divertor Detachment in ASDEX Upgrade
}

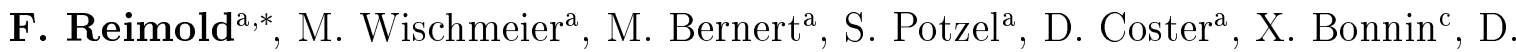
Reiter $^{\mathrm{b}}$, G. Meisl ${ }^{\mathrm{a}}$, A. Kallenbach ${ }^{1}$, L. Aho-Mantila ${ }^{\mathrm{d}}$, U. Stroth ${ }^{\mathrm{a}}$, ASDEX Upgrade Team $^{\mathrm{a}}$

${ }^{a}$ Max-Planck-Institut für Plasmaphysik, Boltzmannstraße 2, D-85748 Garching,Germany

${ }^{b}$ Institut für Energie- und Klimaforschung - Plasmaphysik, Forschungszentrum Jülich GmbH

${ }^{c}$ CNRS-LSPM, Université Paris 13, Sorbonne Paris Cité, F-93430 Villetaneuse, France ${ }^{d}$ VTT, FI-02044 VTT,Finland

\begin{abstract}
Power exhaust in future fusion devices is critical and operation with a detached divertor is forseen for ITER and DEMO. The evolution of detachment in nitrogen seeded H-mode discharges at ASDEX Upgrade is categorized in four phases. Complete detachment of the outer target is found to be correlated with a strongly localized radiation at the Xpoint and a pressure loss at the pedestal top at almost constant core plasma pressure. SOLPS modeling shows that enhanced radial transport in the divertor region is necessary to reconcile the experimental profiles with the simulations. The modeling supports the experimental observation of the correlation of complete detachment with an X-point radiation and a reduction of the pedestal top pressure. A remaining discrepancy are significantly lower neutral densities in the divertor compared to experiment. The effects of wall pumping, the particle reflection model and the boundary conditions on the plasma solution are discussed.
\end{abstract}

Keywords: PACS: 52.55.Rk, 52.25.Xz, 52.25.Vy, 52.55.Fa

PSI-21: Detachment, Nitrogen, Edge modeling, ASDEX-Upgrade, Divertor plasma

\footnotetext{
${ }^{*}$ Corresponding author

Email address: Felix.Reimold@ipp.mpg.de (F. Reimold)
} 


\section{Introduction}

Power exhaust in future fusion devices like ITER [1] and DEMO [2] at reactor relevant fusion power will be a challenge. The expected unmitigated power flux to the divertor target of about $24 \mathrm{MWm}^{-2}$ for ITER [3] already exceeds the specified steady-state material limit of 5 and $10 \mathrm{MWm}^{-2}$ for DEMO [4] and ITER [5] respectively. Therefore, power needs to be dissipated in the scrape-off layer, SOL, by radiation from low-Z impurities like nitrogen and a detached divertor is considered a requirement for target protection in ITER and DEMO [6]. A good experimental characterization and understanding of divertor detachment in H-mode plasmas is critical in order to test the existing theoretical models for detachment [7] and numerical tools like SOLPS [8]. Only a detailed comparison of modeling and experiment can reveal missing physics [9, 10] and help improve the theoretical models and the numerical description of the processes that are relevant in the SOL and the divertor.

Experimental investigations of nitrogen induced detachment at high radiated power fractions, $f_{\text {rad }}$, in H-modes have been carried out at ASDEX Upgrade, AUG. The experimental investigations have been complemented by closely validated modeling with SOLPS5.0.

The experimental setup of the H-mode detachment studies presented in Ref. [11] is characterized and the observations during the detachment evolution are summarized in Section 2, The SOLPS modeling of this experiments is shown in Section 3. Section 4 discusses the observations in the experiment and the modeling and summarizes the paper.

\section{Experiment}

The presented H-mode discharge (\#28903) was run in a lower single null configuration with a plasma current of $I_{\mathrm{P}}=800 \mathrm{kA}$ and a toroidal field of $B_{\mathrm{t}}=2.5 \mathrm{~T}$, which sets the safety factor to $q_{95} \approx 5.5$. The $\nabla B$-drift points into the lower divertor. The total heating power was fixed at 8.2 MW including constant central electron cyclotron resonance heating of $600 \mathrm{~kW}$, neutral beam heating of $7.4 \mathrm{MW}$ and ohmic heating. The deuterium 
fueling and nitrogen seeding rate as well as the main plasma parameters are shown in Figure 1. $\mathrm{a}+\mathrm{b}$. The core plasma nitrogen concentration is derived from charge exchange recombination spectroscopy measurements. It increases from $0.5 \%$ in the non-seeded reference phase to $2.5 \%$ during seeding. The tungsten concentration varies between $c_{\mathrm{W}}=\frac{n_{\mathrm{W}}}{n_{\mathrm{e}}}=2.5-5.0 \times 10^{-5}$.

Figure 1. $\mathrm{c}+\mathrm{d}$ show the evolution of the sum of the ion saturation current density measurements, $j_{\mathrm{sat}}$, to the lower inner and outer target, $I_{\mathrm{sat}}=\sum_{i} j_{\mathrm{sat}, \mathrm{i}}$. For comparison the Two-Point Model scaling of $I_{\text {sat }}$ is also plotted [12]. The scaling is given by $I_{\text {sat,TPM }}=$ $c_{\mathrm{t}_{0}} \bar{n}_{\mathrm{e}, \text { edge }}^{2} P_{\mathrm{H}}^{-\frac{3}{7}}$, where $P_{\mathrm{H}}$ is the heating power, $\bar{n}_{\mathrm{e}, \text { edge }}$ is a line integrated edge density and $c_{\mathrm{t}_{0}}$ is a normalization constant chosen such that $I_{\text {sat }}=I_{\text {sat,TPM }}$ at the reference time, $t_{0}=0.9 \mathrm{~s}$ for the inner target and $t_{0}=2.3 \mathrm{~s}$ for the outer target. Neglecting convective parallel transport, $I_{\text {sat }}<I_{\text {sat,TPM }}$ indicates a pressure loss along fieldlines, i.e. detachment.

The definition of the detachment terms follows Ref. [11. The term partial detachment is defined as a parallel pressure loss at and close to the strikepoint. Complete detachment is defined as strong parallel pressure loss across a large portion of the target $(\Delta S \approx$ $10-15 \mathrm{~cm}$ ) and close to flat target profiles of the ion saturation current and the electron temperature. During complete detachment the peak heat and particle fluxes are reduced by more than one order of magnitude compared to attached conditions.

The time evolution of detachment in nitrogen seeded discharges can be divided into four distinct phases, as indicated in Figure 1.c-e. A more detailed experimental characterization is presented in Ref. [11].

I. Onset of Detachment: Even without impurity seeding $I_{\text {sat }}$ to the inner target drops below the TPM-scaling at $0.9 \mathrm{~s}$ and the inner target detaches inter-ELM. The outer target stays attached.

II. Fluctuating state: At $1.5 \mathrm{~s}$ the inner target is inter-ELM detached and radiative fluctuations at the X-point with a frequency of $6-8 \mathrm{kHz}$ appear in between ELMs [13]. III. Partial detachment of outer target: Nitrogen seeding is applied from $2.8 \mathrm{~s}$ on. The outer target detaches partially during inter-ELM phases. From $3.6 \mathrm{~s}$ the fluctuation frequency decreases until they disappear at $4.0 \mathrm{~s}$ [14]. The ELM size decreases considerably. 
Concomitantly, the bolometers measure the emergence of a strong radiation at the Xpoint with radiated power fractions of $f_{\text {rad }} \approx 85 \%$. An increase in the line integrated density of typically $10-20 \%$ is observed. The inner target is completely detached along the vertical target component.

IV. Complete Detachment: At $3.6 \mathrm{~s}$ the X-point radiation is fully developed and moves further into the confined plasma. Strong Balmer $\mathrm{D}_{\delta}$ and NIII-line radiation at the X-point emerges inside the confined plasma. Both targets are completely detached in inter-ELM phases at $5.5 \mathrm{~s}$. Particle and power fluxes in between ELMs are reduced at both targets to values below $5 \times 10^{22} \mathrm{~m}^{-2} \mathrm{~s}^{-1}$ and $0.5 \mathrm{MWm}^{-2}$, respectively. Midplane profiles of the electron density and the electron and ion temperatures show a clear loss of pressure at the pedestal top of about $40-50 \%$ during complete detachment compared to the non-seeded phase. However, the pressure inside $\rho_{\text {pol }}<0.7$ and hence core confinement is only reduced by $10-15 \%$. The confinement according to the $\mathrm{H}_{98}$-scaling ranges from $0.90-0.95$ during complete detachment and 0.90-1.02 in the non-seeded reference phase.

\section{Modeling}

The SOLPS5.0 code package [8] is used for numerical simulations of the presented plasma scenario. SOLPS mainly consists of two coupled codes: B2.5 is a fluid code that solves Braginskii-like equations for the ions (D, C, He, N) and the electrons. Eirene is a Monte-Carlo code that describes kinetic neutrals [15]. Both codes are coupled via source terms.

The discharge \#28903 is modeled for two time slices: First, a high recycling case without nitrogen seeding at $2.4 \mathrm{~s}$. In this case the outer target is in the high recycling regime. Second, a completely detached case with strong nitrogen seeding at $5.5 \mathrm{~s}$. The separatrix electron density is set to $2.5 \times 10^{19} \mathrm{~m}^{-3}$ in both cases. The input power into the simulation domain is $5-6 \mathrm{MW}$.

The target and midplane profiles of such a simulation are shown for the high recycling case in Figure 2 and for the completely detached case in Figure 3. A simultaneous match of the midplane and the outer target profiles $\left(j_{\text {sat }} \& p_{\mathrm{e}}\right)$ has been achieved in the modeling 
for both cases. A match of the inner divertor target profiles at the same time still remains elusive, especially for the high recycling case. The following will concentrate solely on the outer target if not explicitly stated otherwise. In order to achieve the level of agreement depicted in Figure 2. $\mathrm{c}+\mathrm{d}$ and Figure 3. $\mathrm{c}+\mathrm{d}$, an enhanced perpendicular transport in the divertor region was necessary. The increase of the perpendicular transport in the divertor is a factor of 10 for the high recycling case and a factor of 1.3 in the outer divertor and a factor of 10 in the inner divertor for the detached case. The effect of the enhanced perpendicular transport in the divertor on the target solution will be discussed in Section

\section{1}

The pressure profiles upstream and downstream are in good agreement with the experimental data and hence the experimental pressure loss is reproduced. Despite the good match in the electron pressure, the outer target profiles of the electron density and temperature (not shown) can only be matched in the high recycling case. For the completely detached plasma, the experimental outer target profiles, measured by Langmuir probes, show a factor of up to 10 higher temperatures and lower densities compared to the modeled profiles. The modeled peak density and temperature are $4.6 \times 10^{20} \mathrm{~m}^{-3}$ and $0.6 \mathrm{eV}$ respectively. However, the validity of the Langmuir probe analysis of the electron density and temperature is questionable below $T_{\mathrm{e}} \approx 2 \mathrm{eV}[16$

The ratios of the neutral flux measurements of the poloidally distributed ionization gauges is matched using a subdivertor neutral conductance model. Agreement of the modeled fluxes with the ionization gauges that are influenced by a high field side high density region [17] and that are located behind the high-field side limiter could not be achieved. The overall neutral pressures in the divertor are at least a factor of 5 lower in the modeling than in the experiment. This is consistently observed in a comparison of reconstructed Balmer line emission with spectroscopic signals from the divertor volume and of neutral flux measurements by ionization gauges with modeled neutral fluxes in the subdivertor region.

As observed in the experiment, a saturation of the radiated power fractions at $f_{\mathrm{rad}} \approx 85 \%$ is attained in the modeling. The viewing geometry of the bolometers is shown in Figure 4 
along with the modeled radiation distribution of the high recycling case. The bolometer measurements are compared to reconstructed line integrated power densities from the simulations in Figure 5 for the high recycling case and in Figure 6 for the completely detached case. In the completely detached case, agreement within a factor of 2 was obtained for the dominant contribution of the radiation in the proximity of the X-point. The discrepancy of the radiated power in the global bolometer cameras FVC and FHC away from the Xpoint is due to missing contributions of the core radiation in the simulated line integrals. However, the simulations showed a trend to less radiation in the divertor compared to the experiment. The underestimation of radiation in the divertor leg is in line with the underestimation of the neutral density in the divertor as this radiation is dominated by contributions from neutral deuterium. In the high recycling case, the radiation in the divertor legs close to the target is reproduced within a factor of 2 , but the radiation in the divertor volume between the X-point and the divertor targets is underestimated by the code. This is again consistent with the underestimation the neutral density in the divertor.

In order to achieve completely detached target profiles in modeling it is necessary to seed enough nitrogen such that strongly localized radiation in the proximity of the Xpoint occurs. In the simulations this is often linked to the appearance of strong radiation inside the confined plasma and a correlated decrease of the separatrix temperature from $T_{\mathrm{e}, \mathrm{sep}} \approx 100-120 \mathrm{eV}$ to about $50-80 \mathrm{eV}$. With the X-point radiation inside the confined plasma a reduction of the plasma pressure at the pedestal top of about $50 \%$ compared to unseeded conditions occurs. This reduction of the upstream pressure is not to be confused with the parallel pressure loss along the SOL. The large radiation losses at the X-point also induce the build-up of significant temperature variations along closed field lines with temperatures lower than $10 \mathrm{eV}$ at the $\mathrm{X}$-point. This regime is marginally stable in the simulations without drifts and prone to a radiation collapse of the plasma.

It has been found that the recycling model has a significant impact on the impurity distribution and its concentration level in the confined plasma. This will be discussed in Section 3.3 . The sensitivity of the plasma solution to the boundary conditions and the 
reflection model will be investigated in Section 3.2 .

Most important in order to achieve the presented level of agreement was an unconventional assumption on the perpendicular transport in the divertor region, which will be discussed in the next section.

\subsection{Perpendicular Transport}

SOLPS calculates the perpendicular transport of the plasma according to ad-hoc transport coefficients. A radial profile of these is specified at the midplane. The main transport coefficients are the electron heat conductivity, $\chi_{\mathrm{e}}$, the ion heat conductivity, $\chi_{\mathrm{i}}$, and the particle diffusivity, $D$. Identical transport coefficients are assumed for main and impurity ions.

The modeling approach was to first match the upstream profiles via the adaptation of the radial transport coefficient profiles. The profile shape in all simulations is similar with constant coefficients inside the pedestal, a decrease in the pedestal in order to form the steep gradient region and radially increasing coefficients in the SOL. The magnitude of transport coefficients applied can be found in Table 1.

The simulations show that in order to match the experimental target profiles in the high recycling regime as shown in Figure 2, $\mathrm{c}+\mathrm{d}$ and the completely detached regime as shown in Figure $3 \mathrm{c}+\mathrm{d}$, it is necessary to increase the transport coefficients in the divertor region locally by a factor of 1-10 compared to the SOL above the X-point. The dominant effect of the rescaling is a broadening of the electron density profile around the separatrix and a reduction of the peak density. How the peak particle flux to the target is reduced is different in the inner and outer divertor. The effect of a step-wise increase of the perpendicular transport in the divertor on the target pressure profiles is shown for the inner and outer target in Figure 7. In the inner divertor the temperature drops to low values of about $1 \mathrm{eV}$ across the whole target and the density profile broadens with a reduction of the peak density. Both, the peak pressure and the pressure across the whole target are reduced. In the outer divertor only a peak pressure reduction and a decrease in the peak density with larger fall-off length into the SOL are seen. The peak particle 
flux to the outer target is reduced by a factor of $2-5$, but the integrated flux to the target reduces only by about $10-20 \%$.

\subsection{Boundary conditions}

The boundary conditions at the main chamber and the private flux region boundaries are essential for matching the experimental target data once the radial transport coefficient profile is fixed by the upstream measurements. This section presents the applied boundary conditions and investigates the impact of a variation of these on the plasma solution.

The particle boundary condition at the target is full recycling for all species except for carbon which is pumped. At the core boundary, ions and neutrals "recycle" as fully stripped ions.

At the main chamber and private flux region boundaries, the radial particle flux across the grid boundary of each ion species is specified by $\Gamma_{\mathrm{i}}=\delta n_{\mathrm{i}} c_{\mathrm{s}}$, where $c_{\mathrm{s}}$ is the local sound speed, $n_{\mathrm{i}}$ is the ion density and $\delta$ is a free parameter. The radial particle flux across the grid boundary is recycled at the this boundary as neutrals. The parameter $\delta$ is particularly important in matching the upstream far SOL electron density and hence determines the main chamber recycling level. Values of $\delta=0.01-0.1$ are employed. Wall pumping at the main chamber grid boundary can be mimicked by recycling only a fraction of the flux $\Gamma_{\mathrm{i}}$. For nitrogen the recycling coefficient is $95-100 \%$.

Sheath boundary conditions with separate ion and electron heat transmission coefficients, $\gamma_{\mathrm{e}, \mathrm{i}}$, are applied for energy fluxes at the targets. In line with the literature [18], $\gamma_{\mathrm{e}, \mathrm{i}}=2-3$ reproduce experimental target profiles in attached conditions. In detached conditions the solution is not sensitive in the range $1<\gamma_{\mathrm{e}, \mathrm{i}}<5$. The target parameters change by $20 \%$ at most.

At the main chamber and the private flux region boundaries, temperature fall-off lengths, $\lambda_{\mathrm{e}, \mathrm{i}}$, are specified. Without accurate experimental data, the fall-off lengths are free parameters. A variation in the range $1 \mathrm{~cm}<\lambda_{\mathrm{e}, \mathrm{i}}<10 \mathrm{~cm}$ at the main chamber and private flux region boundaries changes the target solution by less then $5 \%$. The fall-off lengths are not the dominant parameters that determine the power across the grid boundary. 
The power crossing the private flux region grid boundary is mainly set by the available power transported from the common flux region into the private flux region. It is, hence, determined by the transport coefficients in the private flux region. For the main chamber boundary the dominant parameter is $\delta$ as the temperature profiles are rather flat in the far SOL and most of the heat transport is convective. In all simulations the power flux to the main chamber wall is limited to below $1 \mathrm{MW}$.

The core boundary is used to couple the heating power into the simulation domain. A fixed temperature is set at the core boundary in a feedback loop such that the specified power into the domain crosses the core boundary. Equal distribution of the power to electrons and ions is assumed $P_{\mathrm{e}}=P_{\mathrm{i}}=2.5-3.0 \mathrm{MW}$. The power across the core boundary is determined from the experiment by subtracting the radiated power inside the core grid boundary from the experimental heating power.

Momentum boundary conditions have not been varied. At the target sheath boundary conditions enforce the Bohm-Chodura criterion. At all other boundaries the gradient in the radial Mach number profile is set to zero [8].

\subsection{Impurities}

In nitrogen seeded simulations the impurity densities are determined by the recycling location of the impurities and the divertor retention. Additional effort to improve the match of the simulated nitrogen impurity distribution to the experimental measurements with different transport coefficients for main and impurity ions did only result in minor improvements. Sufficiently accurate experimental data to determine transport coefficient profiles for the impurities separately has not been available so far.

A challenge in simulations without drifts at a midplane separatrix density of $n_{\mathrm{e}, \mathrm{sep}}=$ $2.5 \times 10^{19} \mathrm{~m}^{-3}$ is to limit the core impurity content to experimental levels with the seeding rates that are necessary to achieve the experimentally observed radiated power in the completely detached plasma. Main chamber wall pumping of nitrogen reduces the core content of nitrogen due to a lower level of main chamber impurity recycling. It also enhances the code stability for detached conditions with strong radiation at the X-point. 
In line with results from Ref. [19], wall pumping of up to $5 \%$ of the nitrogen flux to the main chamber wall is considered in the simulations.

In modeling parameters studies, the nitrogen core content was shown to rise with decreasing density, increasing heating power and increasing seeding rates. In Figure 8 the thermal and friction forces that act on $\mathrm{N}^{+}$are shown. The larger divertor impurity retention at higher density and lower heating power is mainly due to a dominant increase of friction forces that retain the impurities in the outer divertor, while the counteracting thermal forces are of a similar magnitude in both cases.

\subsection{Particle Reflection Model}

At the inner target the formation of carbon layers by local carbon deposition has been observed in AUG [20]. To assess the possible effect of such carbon layers on the recycling at the target, a change of the inner target material from tungsten to carbon has been performed in the simulations. In the high recycling simulation, the change increases the neutral pressure in the divertor by $20 \%$ and increases the peak density at the inner target by $40 \%$. For detached cases the difference in the peak plasma pressure can be up to a factor of 2 . Additional carbon sources by sputtering were neglected. Hence, the only difference from the change in the target material is in the TRIM reflection coefficients [21] that are used for the fast reflection model of Eirene. This finding points out that it is necessary to accurately determine the particle and energy reflection coefficients and the actual target material composition in the experiments, especially for simulations with low ion impact energies. Additionally, at low impact energies below $50 \mathrm{eV}$ the validity of the binary collision approximation of TRIM gradually breaks down [22. Experimentally benchmarked, more sophisticated molecular dynamics calculations should supplement these data in the future.

\section{Discussion \& Summary}

Experimental H-mode detachment studies [11] showed that the L-mode classification of detachment [23] is valid also in H-mode with the addition of a phase with dominant, 
strongly localized radiation in the proximity of the X-point. Experiments show that the $\mathrm{X}$-point radiation and an associated reduction of the pedestal top pressure are closely correlated to complete detachment in nitrogen seeded H-mode plasmas in AUG.

The X-point radiation and the reduction of the pedestal top pressure can be reproduced in SOLPS simulations. The simulations show that a large reduction of the pedestal top pressure can be induced by the reduction of the temperature near the separatrix due to large parallel energy losses into the radiating volume at X-point inside the confined region. Close to the separatrix significant parallel gradients are then seen in the simulation with temperatures as low as $1 \mathrm{eV}$ on closed field lines close to the $\mathrm{X}$-point.

Modeling also shows that an unconventional assumption of increased perpendicular transport in the divertor is required in simulations of ASDEX Upgrade H-mode plasmas in order to reproduce experimental measurements at the midplane and at the outer target simultaneously.

The applied boundary conditions were within the experimental limits. The simulations showed that the main chamber recycling level of the main ions and impurities are important for determining the far SOL density and the core impurity content respectively. Wall pumping of nitrogen [19] was found to have a significant impact on the core impurity content in simulations without drifts. A change of the particle reflection model at the inner target revealed that the fast particle reflection coefficients can be important, especially in detached plasmas with low temperatures at the target. It is necessary to gather more accurate data from molecular dynamic simulations and experiments in this area in order to improve and constrain the model of the plasma-wall interaction in the simulations.

In summary, it has been demonstrated that new experimental observations during $\mathrm{H}$ mode detachment could be reproduced with SOLPS modeling using an enhanced radial transport in the divertor region. The modeling also supports the experimental observation of the correlation of complete detachment with a dominant, strongly localized radiation in the proximity of the X-point and a pedestal top pressure loss. 


\section{Acknowledgements}

This project has received funding from the Euratom research and training programme $2014-2018$

\section{References}

[1] M Shimada, et al. Nuclear Fusion, 47(6):S1-S17, June 2007.

[2] P. Batistoni. Technical Report CCE-FU 49/6., March 2010.

[3] C. S. Pitcher and P. C. Stangeby. Plasma Phys. Control. Fusion, 39(6):779, June 1997.

[4] A. R. Raffray, et al. Fusion Engineering and Design, 85(1):93-108, January 2010.

[5] A Loarte, et al. Nuclear Fusion, 47(6):S203-S263, June 2007.

[6] A Kallenbach, et al. Plasma Physics and Controlled Fusion, 55(12):124041, December 2013.

[7] P. C Stangeby. The plasma boundary of magnetic fusion devices. Institute of Physics Pub., Bristol; Philadelphia, 2000.

[8] R. Schneider, et al. Contributions to Plasma Physics, 46(1-2):3-191, February 2006.

[9] M. Wischmeier, et al. Journal of Nuclear Materials, 390-391:250-254, June 2009.

[10] L. Aho-Mantila, et al. Journal of Nuclear Materials, 438, Supplement:S321-S325, July 2013.

[11] F. Reimold. Nucl. Fusion, 2014. to be submitted.

[12] A. Loarte, et al. Nucl. Fusion, 38(3):331, March 1998. 00161.

[13] S. Potzel, et al. In EPS 2013, Helsinki, 2013.

[14] S. Potzel, et al. Journal of Nuclear Materials, 438, Supplement:S285-S290, July 2013.

[15] D. Reiter, et al. Fusion Science and Technology, 47(2):172-186, 2005.

[16] M. Weinlich. Technical Report IPP 5/64, Max-Planck Institut for Plasma Physics, Munich, 1995.

[17] S. Potzel. In PSI 2014, Kanazawa, Japan, May 2014.

[18] S. Marsen, et al. Journal of Nuclear Materials, 438:S393-S396, July 2013.

[19] G. Meisl. In PSI 2014, Kanazwa, Japan, May 2014.

[20] M. Mayer, et al. Journal of Nuclear Materials, 390-391:538-543 June 2009.

[21] D. Reiter. Technical Report Jül-2605, Institut für Plasmaphysik Jülich, Association EURATOM KFA, Jülich, Germany, April 1992.

[22] Wolfgang Eckstein. Computer Simulation of Ion-Solid Interactions, volume 10 of Springer Series in Materials Science. Springer Berlin Heidelberg, Berlin, Heidelberg, 1991.

[23] S. Potzel, et al. Nucl. Fusion, 54(1):013001, January 2014. 


\section{Captions}

Figure 1- H-mode detachment experiment \#28903: a - Line integrated density (H1: central, H5: edge) and fueling/seeding rates. b - Heating Power $\left(P_{\text {tot }}\right)$, radiated power $\left(P_{\text {rad }}\right)$ and power to the target $\left(P_{\text {tar }}\right) . \mathrm{c}+\mathrm{d}-$ Inter-ELM, median $I_{\text {sat }},\left\langle I_{\text {sat }}\right\rangle_{\mathrm{ELM}}$, are given in red and blue for the inner and outer target respectively. $I_{\text {sat,TPM }}$ is given in green. e Power spectrogram of AXUV-diode measurement at the X-point.

Figure 2 - High-recycling case of \#28903: Experimental data is shown as grey symbols. SOLPS data is shown in solid and dashed lines for (a) the midplane electron and $\mathrm{N}^{7+}$ density, (b) the midplane electron and ion temperature and (c) the static pressure and (d) the particle flux profile at the outer (OT) and inner (IT) target respectively.

Figure 3- Completely detached case of \#28903: Experimental data is shown as grey symbols. SOLPS data is shown in solid and dashed lines for (a) the midplane electron and $\mathrm{N}^{7+}$ density, (b) the midplane electron and ion temperature and (c) the static pressure and (d) the particle flux profile at the outer (OT) and inner (IT) target respectively.

Figure 4- The LOS geometry of the bolometer system is shown along with the modeled radiation distribution of the high recycling case.

Figure 5- Experimental (black) and reconstructed line integrated power densities for the total radiated power (red) and the radiated power from neutrals (green) are shown for the high recycling.

Figure 6- Experimental (black) and reconstructed line integrated power densities for the total radiated power (red) and the radiated power from neutrals (green) are shown for the completely detached plasma. The divertor bolometer cameras are rescaled by a factor of ten to better compare the shape of the radiation distribution. 
Figure 7 - The effect of a step-wise increase of the perpendicular transport in the divertor on the target pressure profile is shown for the inner (a) and outer (b) divertor in the high recycling simulation. The upstream profiles are not significantly affected and are thus not shown.

Figure 8 - The friction forces and the thermal forces acting on $\mathrm{N}^{+}$ions in the SOL fluxtube next to the separatrix are shown for a low density case (full) and a high density case (dashed). The forces are directed towards the plate for friction and towards the $\mathrm{X}$-point for the thermal force. 


\section{Tables}

Table 1 - The range of transport coefficients for the modeling of \#28903 is given.

\begin{tabular}{|c|c|c|c|}
\hline Quantity & Core & Pedestal & SOL \\
\hline \hline$\chi_{\mathrm{e}}\left[\mathrm{m}^{-2} \mathrm{~s}^{-1}\right]$ & $0.30-0.50$ & $0.01-0.50$ & $0.50-5.00$ \\
\hline$\chi_{\mathrm{i}}\left[\mathrm{m}^{-2} \mathrm{~s}^{-1}\right]$ & $0.30-0.50$ & $0.05-0.50$ & $0.50-5.00$ \\
\hline$D\left[\mathrm{~m}^{-2} \mathrm{~s}^{-1}\right]$ & $0.30-0.70$ & $0.01-0.50$ & $0.50-2.50$ \\
\hline$\alpha$ & $0.00-2.00$ & $0.00-2.00$ & $0.00-2.00$ \\
\hline
\end{tabular}


Figures
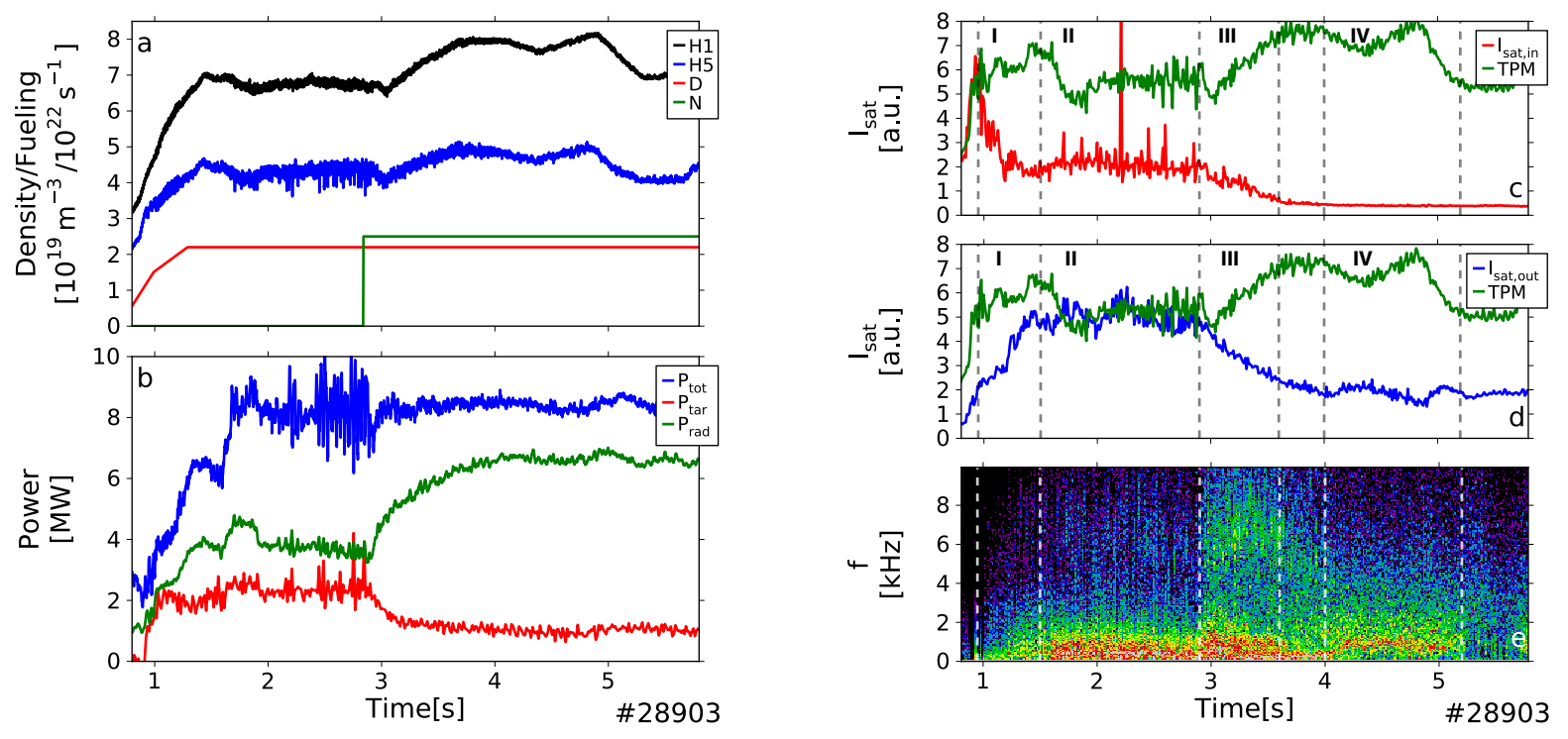

Figure 1 

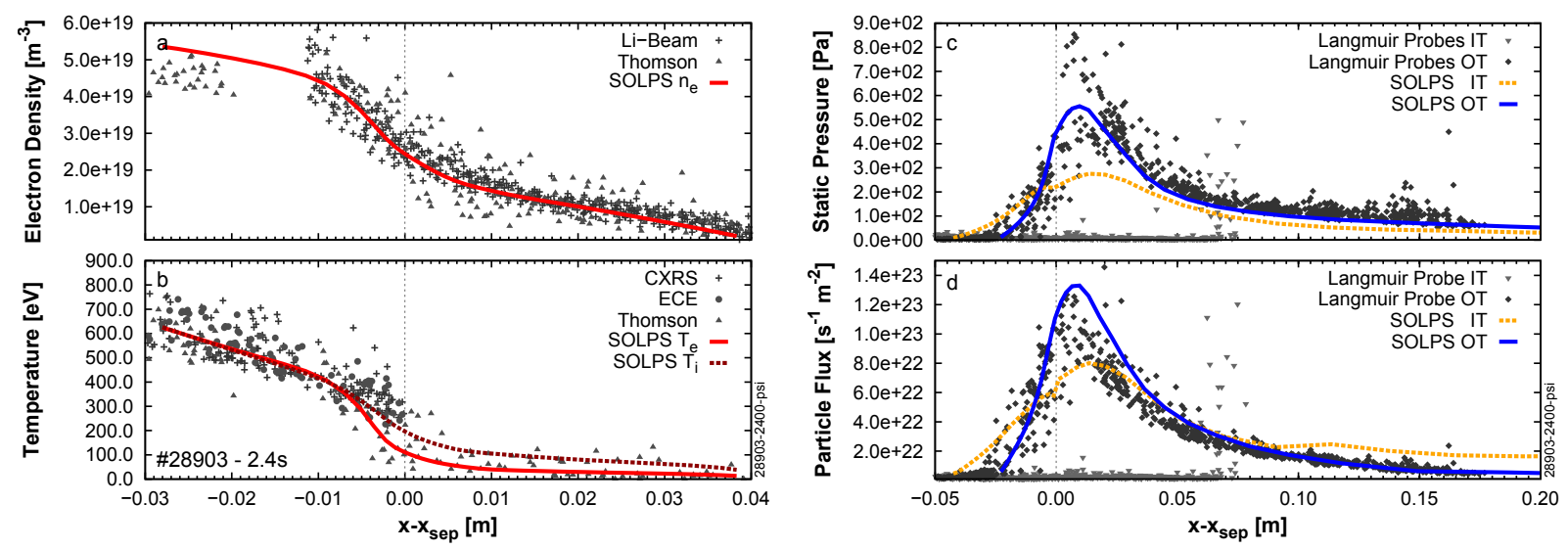

Figure 2 

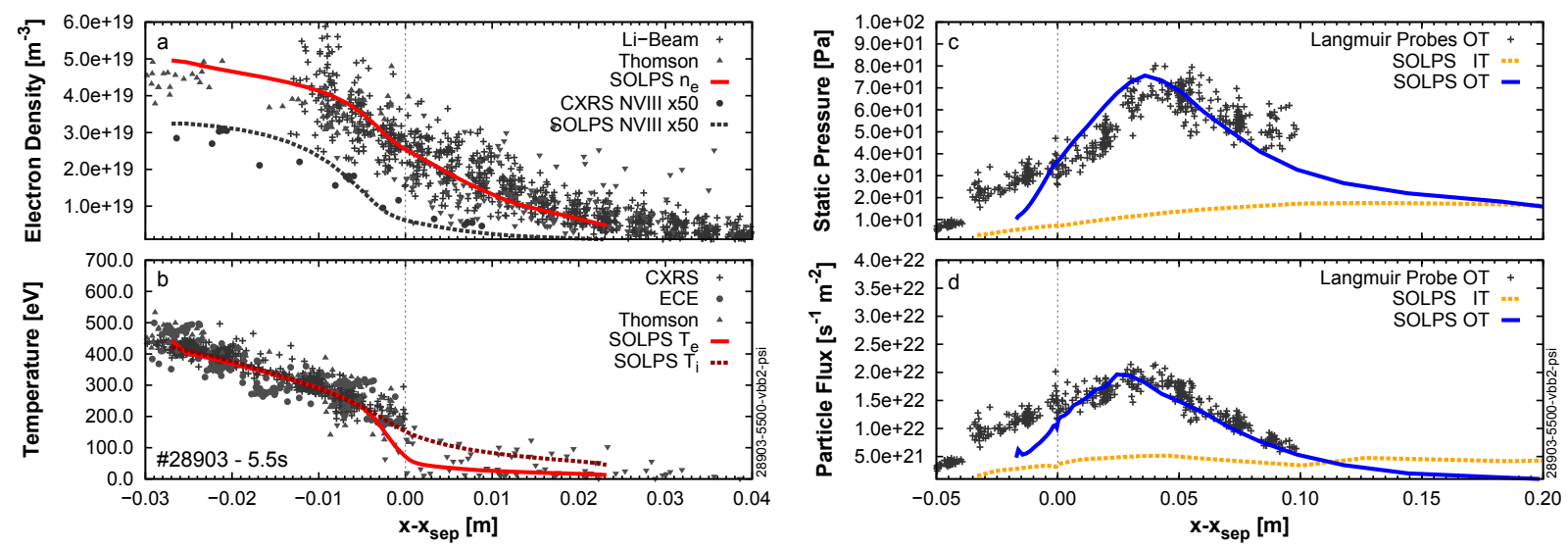

Figure 3 


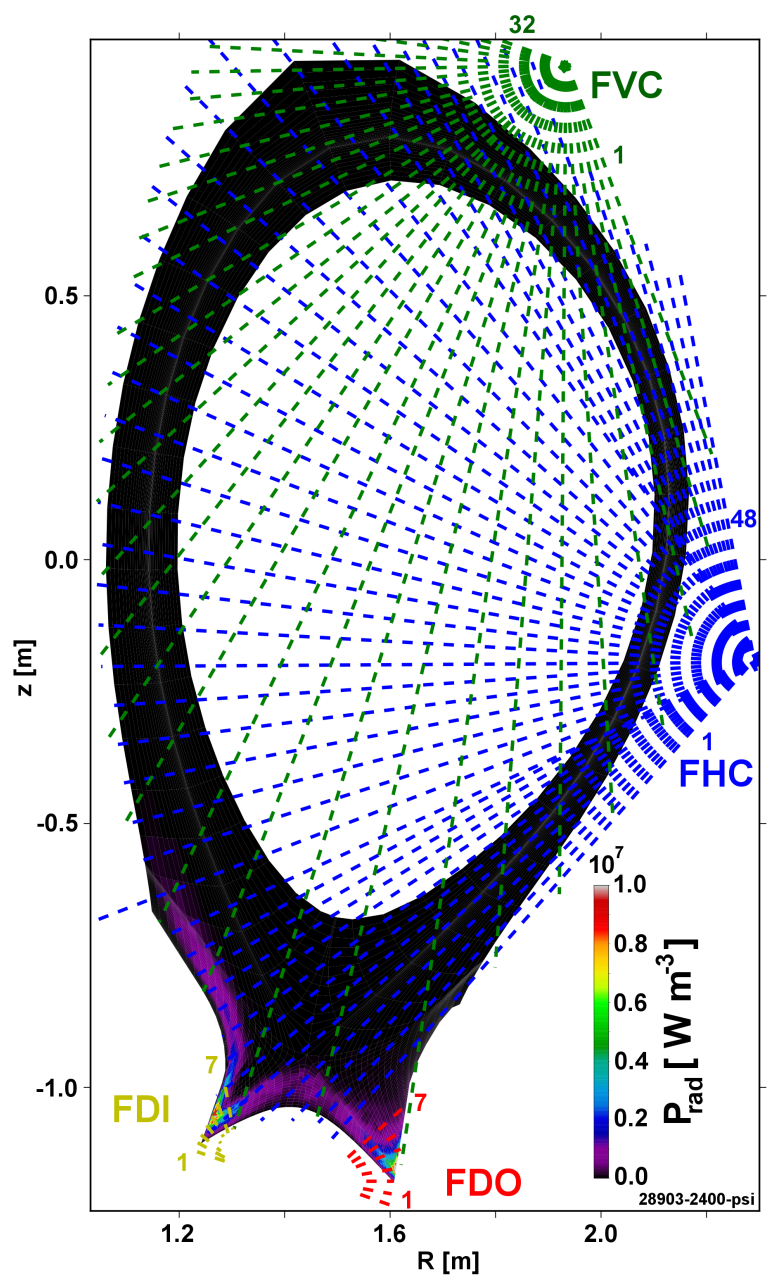

Figure 4 

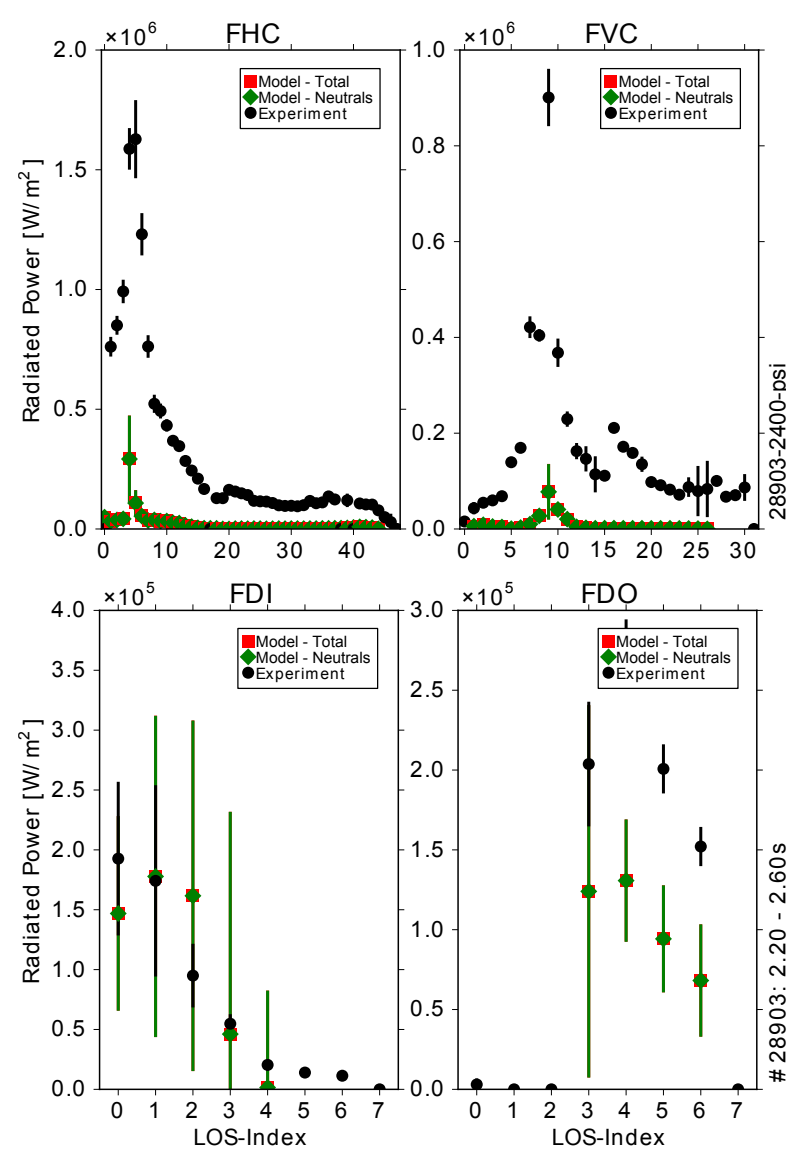

Figure 5 

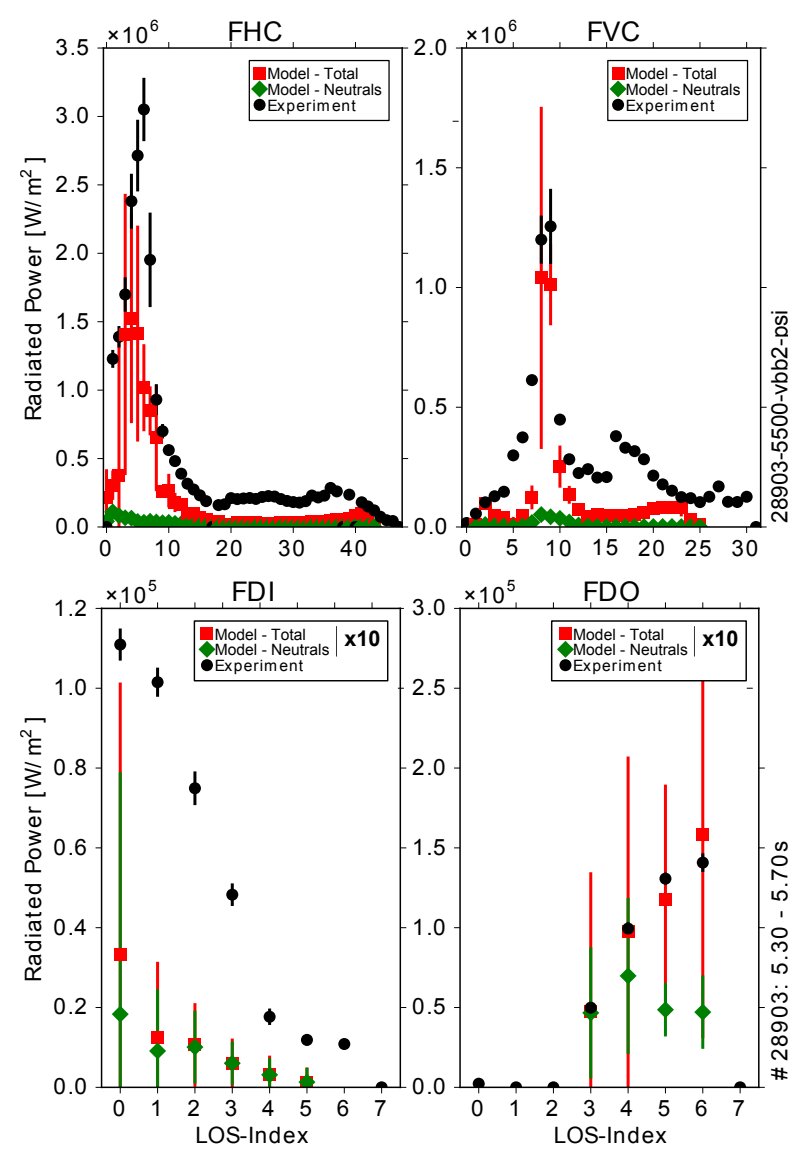

Figure 6 

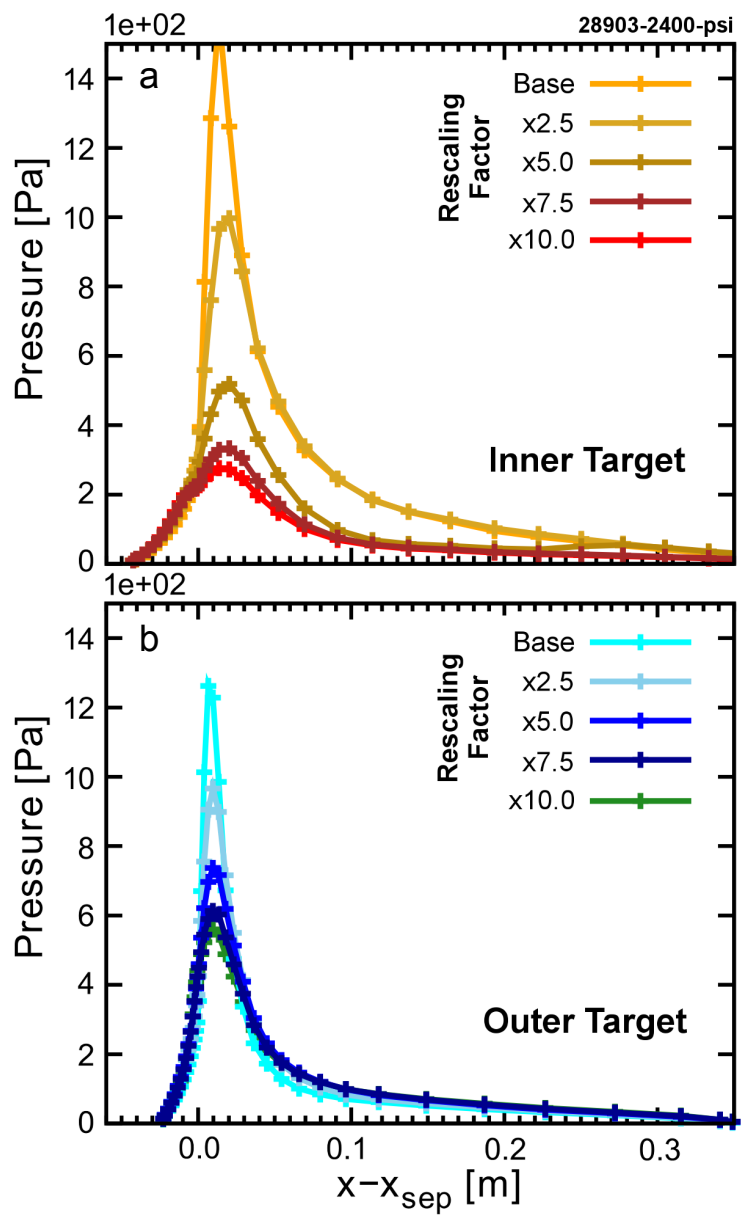

Figure 7 


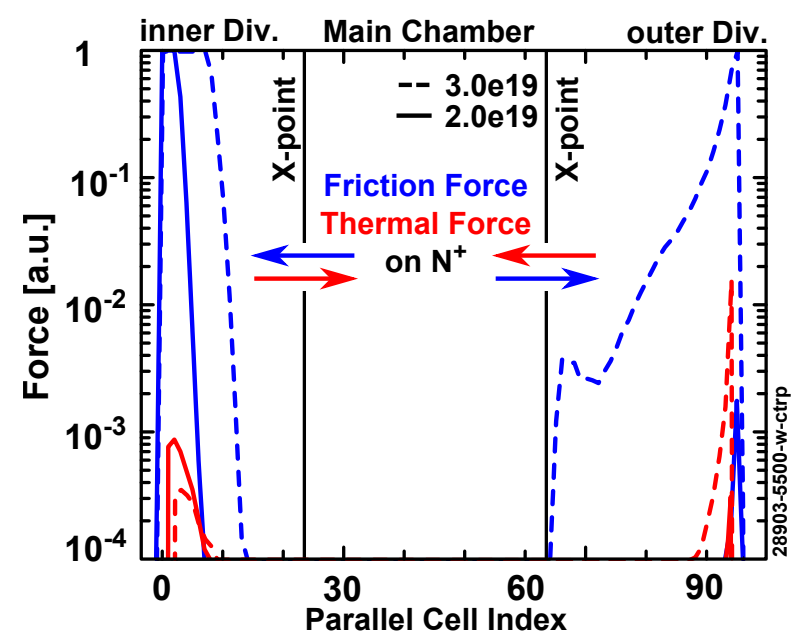

Figure 8 